\title{
Length-dependent conductance and thermopower in single-molecule junctions of dithiolated oligophenylene derivatives: A density functional study
}

\author{
F. Pauly, ${ }^{1,2, *}$ J. K. Viljas, ${ }^{1,2}$ and J. C. Cuevas ${ }^{1,2,3}$ \\ ${ }^{1}$ Institut für Theoretische Festkörperphysik and DFG-Center for Functional Nanostructures, \\ Universität Karlsruhe, 76128 Karlsruhe, Germany \\ ${ }^{2}$ Forschungszentrum Karlsruhe, Institut für Nanotechnologie, 76021 Karlsruhe, Germany \\ ${ }^{3}$ Departamento de Física Teórica de la Materia Condensada, Universidad Autónoma de Madrid, 28049 Madrid, Spain
}

(Received 1 May 2008; published 16 July 2008)

\begin{abstract}
We study theoretically the length dependence of both conductance and thermopower in metal-moleculemetal junctions made up of dithiolated oligophenylenes contacted to gold electrodes. We find that while the conductance decays exponentially with increasing molecular length, the thermopower increases linearly as suggested by recent experiments. We also analyze how these transport properties can be tuned with methyl side groups. Our results can be explained by considering the level shifts due to their electron-donating character as well as the tilt-angle dependence of conductance and thermopower. Qualitative features of the substituent effects in our density functional calculations are explained using a tight-binding model. In addition, we observe symmetry-related even-odd transmission channel degeneracies as a function of molecular length.
\end{abstract}

DOI: 10.1103/PhysRevB.78.035315

PACS number(s): 85.65. + h, 65.80.+n, 73.23.Ad, 73.63.Rt

\section{INTRODUCTION}

In the field of molecular electronics, research has so far mostly concentrated on the dc electrical conduction properties of single-molecule contacts. ${ }^{1}$ By now it is known that the charge transport through organic molecules is typically due to electron tunneling. This is evidenced in particular by the exponential decay of the conductance $G$ with increasing molecular length in contacts formed from oligomers with varying numbers of units. ${ }^{2-4}$ Considering the statistical nature of experiments at the molecular scale, a conclusive comparison to theory is presently difficult. Nevertheless, associated decay coefficients appear to be reproduced by theoretical calculations. ${ }^{5-7}$ For a deeper understanding of molecular-size contacts, it is useful to analyze also other observables in parallel with the dc conductance. Emerging new lines of research involve the photoconductance ${ }^{8-10}$ and heat transport. ${ }^{11,12}$ In this paper we concentrate on the thermopower. For metallic atomic contacts, this quantity was already studied experimentally some years ago ${ }^{13}$ but for molecular contacts only very recently. ${ }^{14,15}$

The thermopower $Q$, also known as the Seebeck coefficient, measures the voltage $\Delta V$ induced over a conducting material at vanishing steady-state electric current $I$, when a small temperature difference $\Delta T$ is applied: $Q$ $=-\Delta V /\left.\Delta T\right|_{I=0}$. It is known that in bulk materials the sign of the thermopower is a hint of the sign of the main charge carriers. If $Q<0(Q>0)$, charge is carried by electronlike (holelike) quasiparticle excitations, as in an $n$-doped ( $p$-doped) semiconductor. ${ }^{16}$ Analogously, the thermopower of the molecular junction gives information about the alignment of the energies of the highest occupied and lowest unoccupied molecular orbitals (HOMO and LUMO) with respect to the metal's Fermi energy $E_{F} \cdot{ }^{17-19}$ In the experiment, ${ }^{14} Q$ was measured for gold electrodes bridged by dithiolated oligophenylene molecules. It was found to be positive, which indicates that $E_{F}$ lies closer to the HOMO than to the LUMO. Also, it was observed that $Q$ grows roughly linearly with the number $N$ of the phenyl rings in the molecule. More recently ${ }^{15}$ the effects of substituents and varied end groups have been analyzed for the benzene molecule.

Using transport calculations based on density functional theory (DFT), we investigate in this paper the length dependence of the conductance and the Seebeck coefficient for dithiolated oligophenylenes bonded to gold contacts. For the molecules studied in Ref. 14, we find that the conductance decays exponentially with increasing molecular length. Decay coefficients compare reasonably with those by previous DFT calculations ${ }^{5-7}$ and also with those from experiments, ${ }^{2-4}$ considering differences in contact configurations, molecular end groups, and uncertainties with respect to environmental effects. In addition, the thermopower increases linearly, with a magnitude coinciding with the measurements. ${ }^{14}$ We also study how the results change as various numbers of methyl substituents are introduced to the molecules. The effect of these substituents is twofold: (i) They push up the energies of the $\pi$ electrons as a result of their electron-donating behavior, ${ }^{15,20}$ and (ii) they increase the tilt angles between the phenyl rings through steric repulsion. The latter effect tends to decrease both $G$ and $Q$ due to a reduction in the degree of $\pi$-electron delocalization, while the former opposes this tendency by bringing the HOMO closer to $E_{F}$. A simplified $\pi$-orbital model is used to explain essential features of the DFT results.

The paper is organized as follows: Section II explains details of our DFT-based approach. Then, in Sec. III, we present the molecular contacts, whose charge transport properties we determine in Sec. IV. In Sec. V we show how the DFT results can be understood in terms of the $\pi$-orbital model, but we discuss also effects beyond this simplified picture in Sec. VI. Finally, we end in Sec. VII with a discussion and conclusions.

\section{METHODS}

The general formulas for the treatment of thermoelectric effects, based on the Landauer-Büttiker formalism, are dis- 


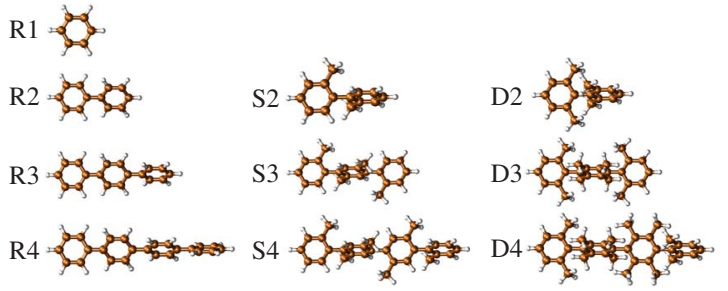

FIG. 1. (Color online) The molecules studied in this work. When they are contacted to the gold electrodes, sulfur atoms replace the terminal hydrogen atoms.

cussed in detail in several references. ${ }^{13,17,19,21-23}$ By expanding the expression for the current $I$ to linear order in $\Delta V$ and $\Delta T$ and considering the cases $\Delta T=0$ and $I=0$, respectively, one arrives at

$$
G=G_{0} K_{0}(T), \quad Q=-\frac{K_{1}(T)}{e T K_{0}(T)},
$$

with $K_{0}(T)=\int \tau(E)[-\partial f(E, T) / \partial E] d E, \quad K_{1}(T)=\int(E-\mu) \tau(E)$ $\times[-\partial f(E, T) / \partial E] d E$, and $G_{0}=2 e^{2} / h$. Here $\tau(E)$ is the transmission function, $f(E, T)=\left\{\exp \left[(E-\mu) / k_{B} T\right]+1\right\}^{-1}$ is the Fermi function, and $\mu$ is the chemical potential, $\mu \approx E_{F}$. At low temperature, the leading-order terms in the Sommerfeld expansions yield

$$
G=G_{0} \tau\left(E_{F}\right), \quad Q=-\frac{k_{B}}{e} \frac{\pi^{2}}{3} \frac{\tau^{\prime}\left(E_{F}\right)}{\tau\left(E_{F}\right)} k_{B} T,
$$

where prime denotes a derivative. In our DFT-based results presented below, we calculate $G$ and $Q$ according to Eq. (1). However, Eq. (2) approximates the results to within a few percent at room temperature, since $\tau(E)$ is smooth around $E_{F}$ due to the off-resonant situation. It should be noted that the equations neglect electron-vibration interactions. These could in principle be included by adding the inelastic corrections to the expression for the current, ${ }^{24}$ but we expect also these contributions to be relatively small even at room temperature.

The transmission functions are computed with the help of Green's-function techniques. The electronic structure is described in terms of DFT as implemented in the quantum chemistry program TURBOMOLE 5.7, where we employ the BP86 exchange-correlation functional and the standard Gaussian basis set of split valence quality with polarization functions on all nonhydrogen atoms. ${ }^{25}$ Geometry optimization is performed until the maximum norm of the Cartesian gradient has decayed to values below $10^{-4}$ a.u. For further details on our method, see Refs. 26-29.

\section{CONTACTS}

The molecules studied are shown in Fig. 1. Those labeled R1-R4 are the pure oligophenylenes. S2-S4 denote oligophenylenes where the hydrogen atom in one of the two ortho positions with respect to each ring-connecting carbon atom is substituted with a methyl group. ${ }^{30,31}$ D2-D4 have substituents in both ortho positions. Here, the numbers $N$ $=1, \ldots, 4$ refer to the number of phenyl rings in the mol-
TABLE I. Geometrical data for the isolated molecules of the R, $\mathrm{S}$, and D series (Fig. 1). The $\varphi_{j}$ are the tilt angles between two adjacent phenyl rings, and $d$ is the distance between the terminal carbon atoms of the molecules.

\begin{tabular}{ccccc}
\hline \hline Molecule & $\begin{array}{c}\varphi_{1} \\
(\mathrm{deg})\end{array}$ & $\begin{array}{c}\varphi_{2} \\
(\mathrm{deg})\end{array}$ & $\begin{array}{c}\varphi_{3} \\
(\mathrm{deg})\end{array}$ & $\begin{array}{c}d \\
(\mathrm{~nm})\end{array}$ \\
\hline R1 & & & & 0.281 \\
R2 & 36.4 & & & 0.718 \\
R3 & 35.3 & 35.4 & & 1.154 \\
R4 & 34.8 & 33.4 & 35.2 & 1.590 \\
S2 & 90.0 & & & 0.715 \\
S3 & 84.8 & 89.8 & & 1.150 \\
S4 & 85.4 & 89.8 & 89.0 & 1.584 \\
D2 & 90.0 & & & 0.715 \\
D3 & 89.1 & 89.2 & & 1.150 \\
D4 & 88.8 & 89.0 & 89.9 & 1.584 \\
\hline \hline
\end{tabular}

ecule. The tilt angles for the R, S, and D molecules vary in the ranges $33.4^{\circ} \leq \varphi_{\mathrm{R}} \leq 36.4^{\circ}, 84.8^{\circ} \leq \varphi_{\mathrm{S}} \leq 90^{\circ}$, and $88.8^{\circ}$ $\leq \varphi_{\mathrm{D}} \leq 90^{\circ}$, and the distances between the terminal carbon atoms of the molecules are described to a good accuracy by $d=a+b N$, with $a=-0.154 \mathrm{~nm}$ and $b=0.435 \mathrm{~nm}$ (Table I). For some of the molecules with two or more rings, different conformational isomers exist. Since for them the absolute values of the tilt angles remain approximately the same, we do not expect essential differences in the results.

The (Kohn-Sham-DFT) HOMO and LUMO energies of the isolated molecules are shown in Fig. 2. We observe that the HOMO-LUMO gaps of the S and D series are larger than those of the R series. Let us note that DFT generally tends to underestimate the gaps. ${ }^{32,33}$

To form the junctions, each molecule is coupled to the hollow position of the tips of two gold [111] pyramids via a sulfur atom. The atomic positions of the molecule and the first gold layers of the tips are then relaxed. This is depicted in Fig. 3 for S3. The relaxed part is also the "central region" in the transport calculations. ${ }^{29}$ The tilt angles $\varphi$ and the distances $d$ of the contacted molecules are not essentially different from those of the isolated molecules.

\section{DENSITY FUNCTIONAL-BASED TRANSPORT}

The results for the transmission, its logarithmic derivative, the conductance, and the thermopower for all of the R, $\mathrm{S}$, and D type molecular junctions are collected in Fig. 4. In

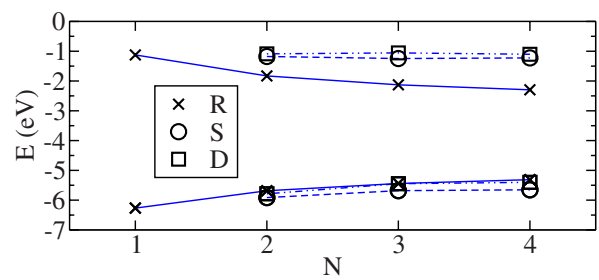

FIG. 2. (Color online) HOMO and LUMO energies of the isolated molecules in Fig. 1. 


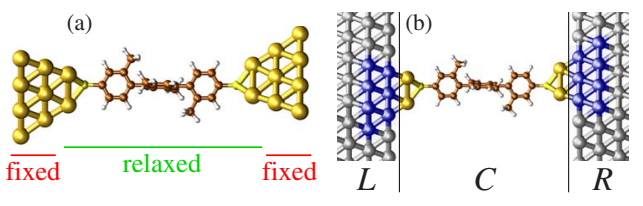

FIG. 3. (Color online) (a) The contacts to the gold electrodes are formed through sulfur atoms bonded to the hollow position of the tip of a [111] pyramid. (b) For the transport calculations, the tips of the pyramids are part of the extended molecule $(\mathrm{C})$. The more remote parts (blue) are absorbed into ideal semi-infinite left (L) and right $(\mathrm{R})$ surfaces (gray).

order to compare with the room-temperature experiments, ${ }^{14}$ we set $T=298 \mathrm{~K}$ in Eq. (1). The Seebeck coefficients are displayed in Fig. 4(d) together with the experimental results, where the molecules R1-R3 were studied.

With increasing number $N$ of phenyl rings, the conductance decays as $G / G_{0} \sim e^{-\beta N}$ [Fig. 4(c)]. For the R series, we find the decay coefficient $\beta_{\mathrm{R}}=1.22,{ }^{8}$ in good agreement with the theory in Ref. $6 .{ }^{34}$ Previous theoretical estimates ${ }^{5,7}$ and experimental results ${ }^{2,3}$ for thiolated self-assembled monolayers are consistently somewhat larger than this value. In particular, the results reported in Ref. 2 vary in the range 1.5 $\leq \beta_{\mathrm{R}} \leq 2.1$, and for amine end groups an experimental value of $\beta_{\mathrm{R}}=1.5$ was reported. ${ }^{4}$ The underestimation of $\beta_{\mathrm{R}}$ may indicate an overestimation of the conductance computed within DFT. ${ }^{35,36}$ However, a comparison between theory and experiment is complicated due to the differences in the end groups used. ${ }^{37}$ For the $\mathrm{S}$ and $\mathrm{D}$ series, we find $\beta_{\mathrm{S}}=3.69$ and $\beta_{\mathrm{D}}=4.07$, which are both much larger than $\beta_{\mathrm{R}}$. This increase reflects the reduced delocalization of the $\pi$-electron system.

Regarding the absolute conductance values, the molecule $\mathrm{R} 1$ has been studied by many groups, and the reported conductances vary mostly between $0.01 G_{0}$ and $0.5 G_{0} \cdot{ }^{38-43}$ Our value of $G_{\mathrm{R} 1}=0.04 G_{0}$ is close to the results in Refs. 41 and 42, for example. The case of R2 is discussed in Ref. 29.

Since the Fermi energy at $E_{F}=-5.0 \mathrm{eV}$ lies closer to the HOMO than to the LUMO level, the Seebeck coefficient $Q$
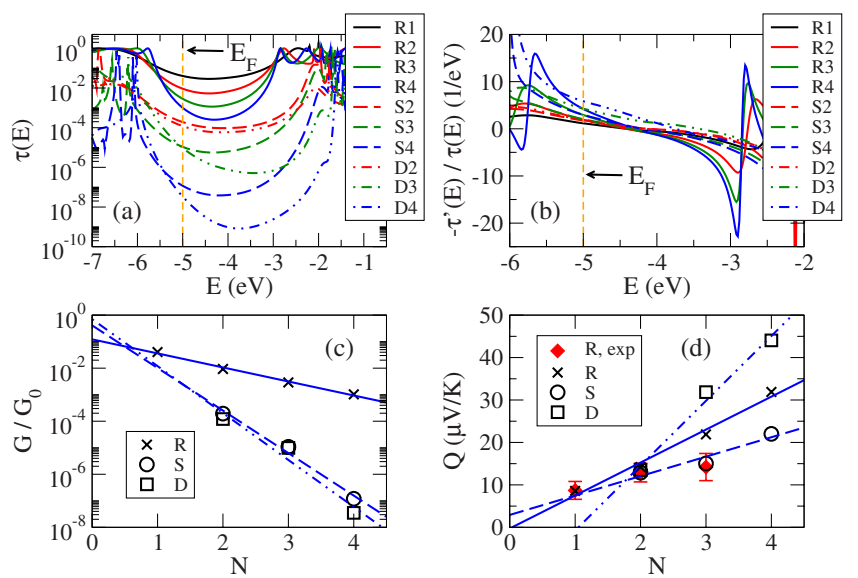

FIG. 4. (Color online) [(a) and (b)] Transmission function and the negative of its logarithmic derivative. [(c) and (d)] The corresponding $G$ and $Q$, including the temperature corrections in Eq. (1). The straight lines are best fits to the numerical results for $\mathrm{R}$ (solid line), S (dashed line), and D (dashed-dotted line). The experimental data in (d) are from Ref. 14. has a positive value [Fig. 4(d)]. We also find that $Q$ increases roughly linearly with $N$, as suggested by the experiments. ${ }^{14}$ Indeed, assuming that the transmission around $E=E_{F}$ is of the form $\tau(E)=\alpha(E) e^{-\beta(E) N}$, then Eq. (2) yields $Q=Q^{(0)}$ $+Q^{(1)} N$, where $Q^{(0)}=-k_{B}^{2} T \pi^{2}\left[\ln \alpha\left(E_{F}\right)\right]^{\prime} / 3 e$ and $Q^{(1)}$ $=k_{B}^{2} T \pi^{2} \beta^{\prime}\left(E_{F}\right) / 3 e .{ }^{44}$ Two things should be noted here. First, $Q$ does not necessarily extrapolate to zero for $N=0$, leading to a finite "contact thermopower" $Q^{(0)}$. Second, $Q^{(0)}$ depends on the prefactor $\alpha(E)$, but $Q^{(1)}$ does not. Since $\alpha(E)$ contains the most significant uncertainties related to the contact geometries, $Q^{(1)}$ can be expected to be described at a higher level of confidence than $Q^{(0)}$. Best fits to our results and the experimental data give $Q_{\mathrm{R}}^{(0)}=-0.28 \mu \mathrm{V} / \mathrm{K}, Q_{\mathrm{R}}^{(1)}$ $=7.77 \mu \mathrm{V} / \mathrm{K}$ and $Q_{\mathrm{R}, \exp }^{(0)}=6.43 \mu \mathrm{V} / \mathrm{K}, Q_{\mathrm{R}, \exp }^{(1)}=2.75 \mu \mathrm{V} / \mathrm{K}$, respectively. Differences in the fit parameters mainly stem from the data point for R3, where the experimental value is lower than the calculated one. Considering the reported order-of-magnitude discrepancies between measured conductances and those computed from DFT, ${ }^{35,36}$ the agreement still appears reasonable. ${ }^{45}$ However, for large enough $T$ and $N$, the above-mentioned exponential and linear laws for the length dependences of $G$ and $Q$ should be modified due to interactions with the thermal environment. ${ }^{19}$ The fact that the experimental data in Fig. 4(d) exhibit a rather good linearity suggests that molecular vibrations do not play a crucial role and that the electronic contribution to $Q$ is dominant.

Although the conductances for S and D are very similar, their thermopowers are rather different. Furthermore, the magnitudes for $N>2$ follow the surprising order $Q_{\mathrm{S}}<Q_{\mathrm{R}}$ $<Q_{\mathrm{D}} \cdot{ }^{46}$ As we will discuss below, these observations can be understood through the two competing substituent effects: (i) a change in the alignment of the $\pi$-electron levels with respect to $E_{F}$ as a result of the electron-donating nature of the methyl group ${ }^{15,20}$ and (ii) an increase in the ring-tilt angles. For the isolated molecules, the second effect results in the opening of the HOMO-LUMO gap when going from molecules $\mathrm{R}$ to $\mathrm{S}$ or $\mathrm{D}$, while the first effect causes the difference between the HOMO and LUMO energies of the S and D series (Fig. 2).

\section{V. $\pi$-ORBITAL MODEL}

In order to understand better the general features of the dependence of $G$ and $Q$ on the number of substituents, we study a simple tight-binding (TB) model, which describes the $\pi$-electron system of the oligophenylenes (Fig. 5). The onsite energies $\epsilon_{j}(j=1, \ldots, N)$ are equal on all carbon atoms of phenyl ring $j$, the intra-ring hopping $t$ is assumed to be the same everywhere, and the inter-ring hopping $u$ is parametrized through $u=t \cos \varphi$. We assume the effect of the side groups to come into play only through $\varphi$ and $\epsilon_{j}$. The leads are modeled by "wide-band" self-energies $\Sigma_{\mathrm{L}, \mathrm{R}}=-i \Gamma$, acting on the terminal carbon atoms. Notice that since $u$ is even in $\varphi$, the model does not distinguish between conformational isomers, where tilt angles change sign.

We extract the parameters of our model as follows: For the R molecules, we set $\epsilon_{j}=0$. In the S and D molecules, the $\epsilon_{j}$ 's for rings with one, two, or four methyl groups are obtained by performing DFT calculations of methylbenzene, dimethylbenzene, and tetramethylbenzene (Fig. 6). We find 


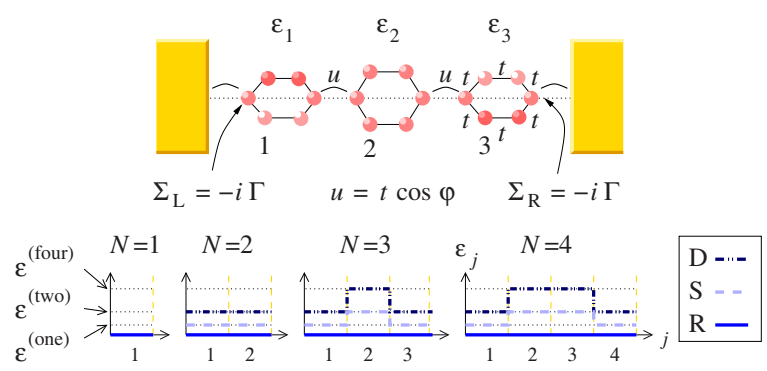

FIG. 5. (Color online) The parameters of the TB model represented by a molecule with $N=3$ phenyl rings. Also shown are schematic graphs of the on-site energies $\epsilon_{j}$ of the different rings $j$ $=1, \ldots, N$ for $\mathrm{R}, \mathrm{S}$, and $\mathrm{D}$ type molecules with $N=1, \ldots, 4$ rings.

that the HOMO and LUMO energies in these molecules shift upward monotonously with the number of methyl substituents. This can be attributed to the electron-donating character of the methyl groups and the resulting increase in Coulomb repulsion on the phenyl ring. We use the averages of the HOMO and LUMO shifts relative to benzene and obtain, respectively, $\epsilon^{\text {(one) }}=0.17 \mathrm{eV}, \epsilon^{(\mathrm{two})}=0.33 \mathrm{eV}$, and $\epsilon^{\text {(four) }}$ $=0.58 \mathrm{eV} \cdot{ }^{47}$ The on-site energies of the different phenyl rings of the $\mathrm{R}, \mathrm{S}$, and $\mathrm{D}$ molecules with $N=1, \ldots, 4$ are schematically represented in the lower part of Fig. 5. The hopping $t$ is set to half of (the negative of) the HOMO-LUMO gap of benzene, with the result $t=-2.57 \mathrm{eV}$ (Fig. 2). The scattering rate $\Gamma$ and the tilt angles for $\mathrm{R}, \mathrm{S}$, and D molecules are chosen to reproduce approximately the minimal transmissions for R1, R2, S2, and D2 in the DFT results in Fig. 4(a). This yields $\Gamma=0.64 \mathrm{eV}, \varphi_{\mathrm{R}}=40.0^{\circ}, \varphi_{\mathrm{S}}=85.5^{\circ}$, and $\varphi_{\mathrm{D}}$ $=86.5^{\circ}$. The last free parameter is $E_{F}$, which should be de-

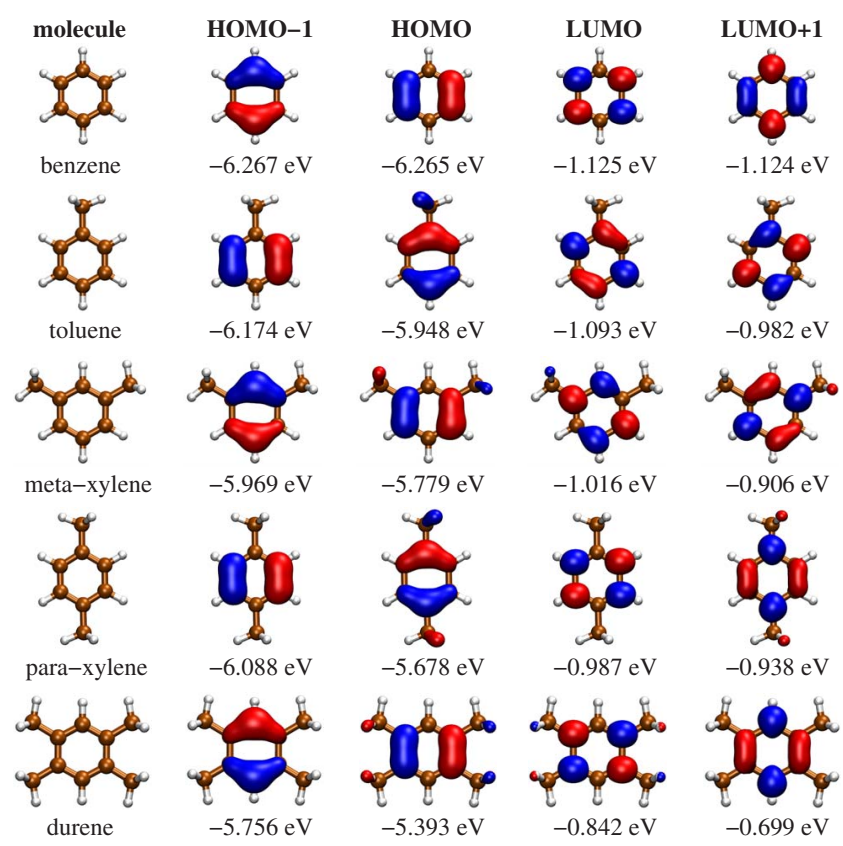

FIG. 6. (Color online) Influence of methyl substituents on the level alignment of frontier molecular orbitals in benzene. From left to right the molecular structure is displayed together with isosurface plots of the HOMO-1, HOMO, LUMO, and LUMO+1 wave functions. Below these plots the common name of the molecules and the energies of the molecular levels are indicated.
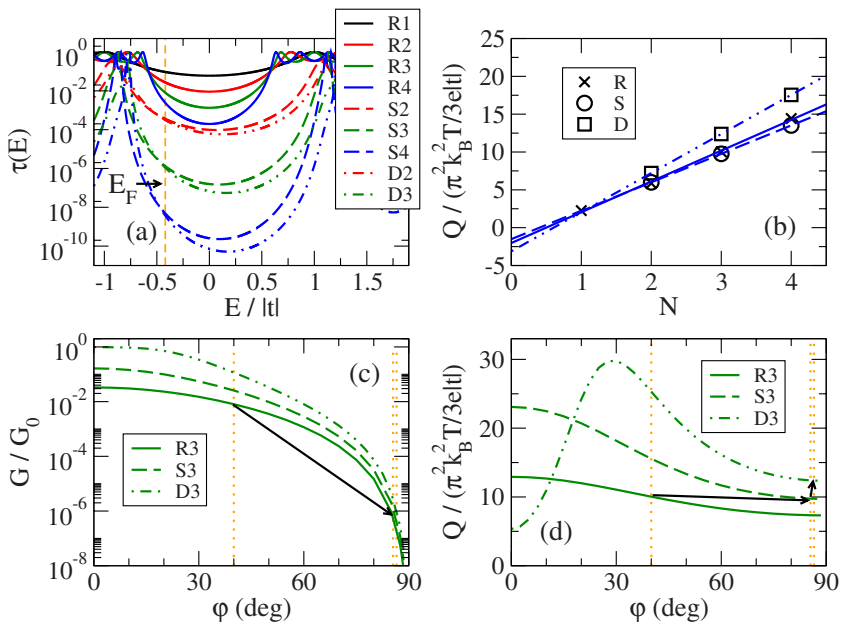

FIG. 7. (Color online) (a) Transmission functions for the TB model in Fig. 5 with parameters chosen as explained in the text. (b) Corresponding Seebeck coefficients according to Eq. (2). The straight lines are best fits as in Fig. 4. [(c) and (d)] $G$ and $Q$ as a function of the tilt angle for the molecules with $N=3$. The vertical dotted lines indicate the "equilibrium angles" $\varphi_{R}, \varphi_{S}$, and $\varphi_{D}$; the arrows represent changes when going from R3 to S3 to D3.

termined by the overall charge-transfer effects between the molecule and the electrodes. We set its value to $E_{F}$ $=-1.08 \mathrm{eV}$, close to the crossing points of the transmission curves of the S and D molecules [cf. Figs. 4(a) and 7(a)].

Although the model is not meant to reproduce all the details of the DFT results, similar features can be recognized. In particular, as shown in Fig. 7(b), the correct order of the thermopowers is reproduced. In going from $\mathrm{R}$ to $\mathrm{S}$, the increase in the tilt angle and the associated breaking of the $\pi$-electron conjugation dominates over other side group effects. As a result both $G$ [Fig. 7(c)] and $Q$ [Fig. 7(d)] decrease. In going from $\mathrm{S}$ to $\mathrm{D}$, an interplay between the sidegroup-induced level shifts and a small residual increase in $\varphi$ raise $Q$ above the value for $\mathrm{R}$, while $G$ remains almost unchanged. We note that for an $N$-ring junction, the lowestorder terms in an expansion of the transmission in powers of $u$ yield $\varphi$ dependences of the form $\tau(E) \approx c_{1}(E) \cos ^{2(N-1)} \varphi$ $+c_{2}(E) \cos ^{2 N} \varphi$, for $E \approx E_{F}{ }^{29}$ Independently of $N$, this results in $Q \approx q_{1}+q_{2} \cos ^{2} \varphi$, where we find $q_{1}, q_{2}>0$ in our case. For $N=2$, the neglect of the $c_{2}$ term leads to the well-known " $\cos ^{2} \varphi$ law" of $G,{ }^{4,29}$ but we see that for the tilt-angle dependence of $Q$ the presence of this term is significant, since $q_{2}=0$ if $c_{2}=0$. When $E_{F}$ is close to a resonance, further higher-order terms become increasingly important and deviations from the $q_{1}+q_{2} \cos ^{2} \varphi$ law result. This is seen most clearly as the nonmonotonous $\varphi$ dependence of $Q$ for molecule D3 in Fig. 7(d).

\section{EFFECTS BEYOND THE $\pi$-ORBITAL MODEL}

Close to perpendicular ring tilts, results from the $\pi$-orbital model should be considered with care. At $\varphi=90^{\circ}$ the $\pi$ - $\pi$ coupling $u$ vanishes, and any other couplings between the rings will become important. ${ }^{48}$ Let us analyze this for the biphenyl molecules R2, S2, and D2. For them, we have varied the tilt angle between the rings and have determined the 

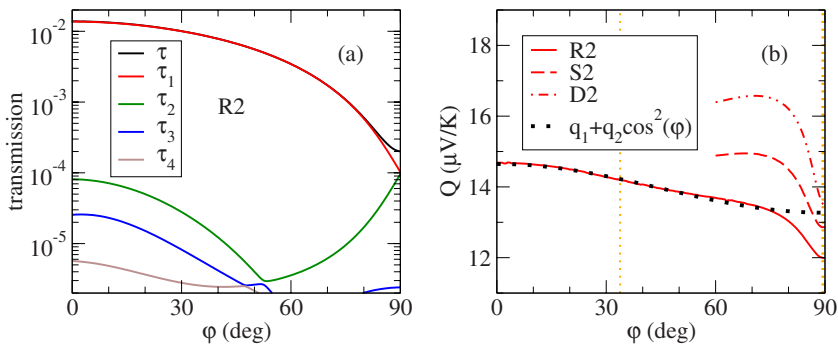

FIG. 8. (Color online) (a) Tilt-angle-dependent transmission $\tau$ $=\Sigma_{i} \tau_{i}$ resolved in its transmission channels $\tau_{i}$ for the molecule R2 and (b) thermopower for R2, S2, and D2. The curve for R2 is fitted by a function of the form $q_{1}+q_{2} \cos ^{2} \varphi$ with $q_{1}=13.27 \mu \mathrm{V} / \mathrm{K}$ and $q_{2}=1.38 \mu \mathrm{V} / \mathrm{K}$ for the tilt-angle interval from $0^{\circ}$ to $60^{\circ}$. The dotted vertical lines indicate DFT equilibrium tilt angles $\varphi_{\mathrm{R} 2}, \varphi_{\mathrm{S} 2}$, and $\varphi_{\mathrm{D} 2}$.

charge transport properties for every $\varphi$ by using our DFTbased approach. Further details of the procedure are described in Ref. 29. For R2 (and similarly for S2 and D2) we observe that for most tilt angles $\left(\varphi \lesssim 80^{\circ}\right)$ the transmission is dominated by a single channel [Fig. 8(a)] of $\pi-\pi$ character. However, at large angles two transmission channels of the same magnitude are observed, which become degenerate at $\varphi=90^{\circ}$. They arise from $\sigma-\pi$ couplings between the two rings, whose strengths are proportional to $\sin \varphi$. Thus the two degenerate channels are of the $\sigma-\pi$ and $\pi-\sigma$ types. These features are obviously not accounted for by the TB model, where only a single $\pi-\pi$ channel is present independently of $\varphi$. The $\sigma-\pi$ couplings should also modify the tiltangle dependence of the thermopower, which is plotted in Fig. 8(b). Due to the electron-donating nature of the methyl groups, the thermopower at fixed $\varphi$ increases from R2 to S2 and D2. While the curve for R2 can be described by the law $q_{1}+q_{2} \cos ^{2} \varphi$ for $\varphi \leqq 80^{\circ}$, a dip is observed for larger $\varphi$. Similar deviations in $Q$ are also present for S2 and D2, where we have investigated a smaller tilt-angle interval because of the steric repulsion of the methyl groups. ${ }^{29}$

The degeneracy of the transmission channels is due to the $D_{2 d}$ symmetry of biphenyl when $\varphi=90^{\circ} .{ }^{48}$ For the longer oligophenylenes, the symmetry $D_{2 d}$ can occur if and only if tilt angles are all at $90^{\circ}$ and the number of rings is even. Hence for S2, S4, D2, and D4, the ratio $\tau_{2} / \tau_{1}$ of the first two transmission channels should be particularly large. Such even-odd oscillations are indeed visible in Fig. 9 for the $S$ and $\mathrm{D}$ series, while they are absent for R. Owing to the fact

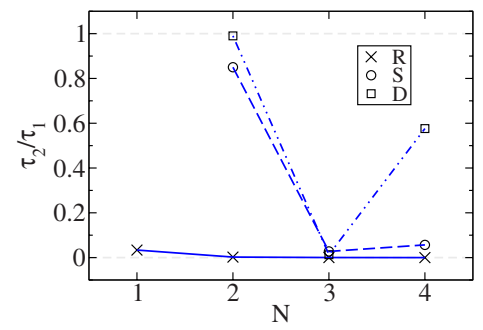

FIG. 9. (Color online) Importance $\tau_{1} / \tau_{2}$ of the second transmission channel $\tau_{2}$ as compared to the first $\tau_{1}$ as a function of molecular length for the molecules of series R, S, and D. that tilt angles deviate from $90^{\circ}$, the oscillations decay. In particular $\tau_{2} / \tau_{1}$ is much smaller for S4 than for D4, where the minimal tilt angles in the contacts are $85.1^{\circ}$ and $89.1^{\circ}$, respectively.

\section{DISCUSSION AND CONCLUSIONS}

As is well known, there are many theoretical uncertainties involved in the determination of transport properties based on DFT calculations; improvements for the methods of molecular-scale transport theory are currently being sought. ${ }^{32,35,36}$ Indeed, it is typical to find order-of-magnitude differences between measured conductances and those computed within DFT. ${ }^{35,36}$ It is also known that atomic configurations can have a strong influence on the conductance. ${ }^{36,49,50}$ Hence a conclusive comparison with experimental data would require the statistical analysis of a large number of contact geometries. However, models predict $Q$ to be insensitive to changes in the lead couplings: ${ }^{17}$ hence it is expected to be a more robust quantity than $G$. Although the measurements in Ref. 14 were carried out at room temperature, we do not expect molecular vibrations to play an essential role in the results due to the weakness of the electron-vibration coupling. Thus, the comparison we have made with our elastic transport theory seems justified. Nevertheless, the investigation of the effect of molecular vibrations is an interesting direction for future research, also in view of optimizing the properties of molecular thermoelectric devices for potential applications. As far as gaining a basic understanding is concerned, low-temperature measurements would be desirable to remove uncertainties related to the thermal excitation of vibrations. The purpose of the present work was to study the general trends for a series of molecules that are similarly coupled to the electrodes. Considering all the potential uncertainties, the agreement we obtain for the thermopower appears to be quite reasonable.

In conclusion, we have analyzed the length dependence of conductance and thermopower for oligophenylene singlemolecule contacts. While we found the conductance to decay exponentially with the length of the molecule, we observe that the thermopower increases linearly. For possible future applications, it is interesting to know how the magnitudes of these quantities can be tuned. We analyzed how this can be achieved by chemical substituents, which control the Fermilevel alignment and the molecular conformation. We demonstrated that a simple $\pi$-electron tight-binding model can help to understand basic substituent effects. In addition, we observed an even-odd effect for transmission channel degeneracies upon variation of the number of phenyl rings, which we explained by molecular symmetries.

\section{ACKNOWLEDGMENTS}

We acknowledge stimulating discussions with M. Mayor. The Quantum Chemistry group of R. Ahlrichs is thanked for providing us with TURBOMOLE. This work was financially supported by the Helmholtz Gemeinschaft (Contract No. VH-NG-029), the EU network BIMORE (Grant No. MRTNCT-2006-035859), and DFG SPP 1243. F.P. acknowledges the funding of a Young Investigator Group at KIT. 
*fabian.pauly@kit.edu

${ }^{1}$ N. J. Tao, Nat. Nanotechnol. 1, 173 (2006).

${ }^{2}$ D. J. Wold, R. Haag, M. A. Rampi, and C. D. Frisbie, J. Phys. Chem. B 106, 2813 (2002).

${ }^{3}$ S. Wakamatsu, S. Fujii, U. Akiba, and M. Fujihara, Jpn. J. Appl. Phys., Part 1 45, 2736 (2006).

${ }^{4}$ L. Venkataraman, J. E. Klare, M. S. Hybertsen, and M. L. Steigerwald, Nature (London) 442, 904 (2006).

${ }^{5}$ C.-C. Kaun, B. Larade, and H. Guo, Phys. Rev. B 67, 121411(R) (2003).

${ }^{6}$ M. Kondo, T. Tada, and K. Yoshizawa, J. Phys. Chem. A 108 , 9143 (2004)

${ }^{7}$ W. Su, J. Jiang, and Y. Luo, Chem. Phys. Lett. 412, 406 (2005).

${ }^{8}$ J. K. Viljas, F. Pauly, and J. C. Cuevas, Phys. Rev. B 76, 033403 (2007).

${ }^{9}$ D. C. Guhr, D. Rettinger, J. Boneberg, A. Erbe, P. Leiderer, and E. Scheer, Phys. Rev. Lett. 99, 086801 (2007).

${ }^{10}$ J. K. Viljas and J. C. Cuevas, Phys. Rev. B 75, 075406 (2007).

${ }^{11}$ M. Galperin, A. Nitzan, and M. A. Ratner, Phys. Rev. B 75, 155312 (2007).

${ }^{12}$ Z. Wang, J. A. Carter, A. Lagutchev, Y. K. Koh, N.-H. Seong, D. G. Cahill, and D. D. Dlott, Science 317, 787 (2007).

${ }^{13}$ B. Ludoph and J. M. van Ruitenbeek, Phys. Rev. B 59, 12290(R) (1999).

${ }^{14}$ P. Reddy, S.-Y. Jang, R. A. Segalman, and A. Majumdar, Science 315, 1568 (2007)

${ }^{15}$ J. A. M. K. Baheti, P. Doak, P. Reddy, S.-Y. Jang, T. D. Tilley, A. Majumdar, and R. A. Segalman, Nano Lett. 8, 715 (2008).

${ }^{16}$ N. W. Ashcroft and N. D. Mermin, Solid State Physics (Saunders, New York, 1976).

${ }^{17}$ M. Paulsson and S. Datta, Phys. Rev. B 67, 241403(R) (2003).

${ }^{18}$ J. Koch, F. von Oppen, Y. Oreg, and E. Sela, Phys. Rev. B 70, 195107 (2004).

${ }^{19}$ D. Segal, Phys. Rev. B 72, 165426 (2005).

${ }^{20}$ L. Venkataraman, Y. S. Park, A. C. Whalley, C. Nuckolls, M. S. Hybertsen, and M. L. Steigerwald, Nano Lett. 7, 502 (2007).

${ }^{21}$ U. Sivan and Y. Imry, Phys. Rev. B 33, 551 (1986).

${ }^{22}$ B. Wang, Y. Xing, L. Wan, Y. Wei, and J. Wang, Phys. Rev. B 71, 233406 (2005).

${ }^{23}$ H. van Houten, L. W. Molenkamp, C. W. J. Beenakker, and C. T. Foxon, Semicond. Sci. Technol. 7, B215 (1992).

${ }^{24}$ J. K. Viljas, J. C. Cuevas, F. Pauly, and M. Häfner, Phys. Rev. B 72, 245415 (2005).

${ }^{25}$ R. Ahlrichs, M. Bär, M. Häser, H. Horn, and C. Kölmel, Chem. Phys. Lett. 162, 165 (1989).

${ }^{26}$ S. Wohlthat, F. Pauly, J. K. Viljas, J. C. Cuevas, and G. Schön, Phys. Rev. B 76, 075413 (2007).
${ }^{27}$ F. Pauly, Ph.D. thesis, Universität Karlsruhe (TH), 2007.

${ }^{28}$ F. Pauly, J. K. Viljas, U. Huniar, M. Häfner, S. Wohlthat, M. Bürkle, J. C. Cuevas, and G. Schön, arXiv:0806.4173 (unpublished).

${ }^{29}$ F. Pauly, J. K. Viljas, J. C. Cuevas, and G. Schön, Phys. Rev. B 77, 155312 (2008).

${ }^{30}$ M. Elbing, Ph.D. thesis, Forschungszentrum Karlsruhe, 2005.

${ }^{31}$ A. Shaporenko, M. Elbing, A. Błaszcyk, C. von Hänsch, M. Mayor, and M. Zharnikov, J. Phys. Chem. B 110, 4307 (2006).

${ }^{32}$ K. S. Thygesen and A. Rubio, Phys. Rev. B 77, 115333 (2008).

${ }^{33}$ R. W. Godby and P. García-González, in A Primer in Density Functional Theory, edited by C. Fiolhais, F. Nogueira, and M. Marques (Springer, Berlin, 2003).

${ }^{34}$ The decay coefficient reported in Ref. 6 for the $\mathrm{R}$ series is $2.56 \mathrm{~nm}^{-1}$ as compared to our value of $\beta_{\mathrm{R}} / b=2.80 \mathrm{~nm}^{-1}$.

${ }^{35}$ C. Toher, A. Filippetti, S. Sanvito, and K. Burke, Phys. Rev. Lett. 95, 146402 (2005).

${ }^{36}$ S. Y. Quek, L. Venkataraman, H. J. Choi, S. G. Louie, M. S. Hybertsen, and J. B. Neaton, Nano Lett. 7, 3477 (2007).

${ }^{37}$ In the experiments with thiols (Refs. 2 and 3), molecules bind asymmetrically only to one side of the electrode, and in Ref. 4 diamines were used.

${ }^{38}$ S. V. Faleev, F. Léonard, D. A. Stewart, and M. van Schilfgaarde, Phys. Rev. B 71, 195422 (2005).

${ }^{39}$ C. Toher and S. Sanvito, Phys. Rev. Lett. 99, 056801 (2007).

${ }^{40}$ M. Strange, I. S. Kristensen, K. S. Thygesen, and K. W. Jacobsen, J. Chem. Phys. 128, 114714 (2008).

${ }^{41}$ Y. Xue and M. A. Ratner, Phys. Rev. B 68, 115406 (2003).

${ }^{42}$ K. Varga and S. T. Pantelides, Phys. Rev. Lett. 98, 076804 (2007).

${ }^{43}$ K. Stokbro, J. Taylor, M. Brandbyge, J.-L. Mozos, and P. Ordejón, Comput. Mater. Sci. 27, 151 (2003).

${ }^{44}$ J. K. Viljas, F. Pauly, and J. C. Cuevas, Phys. Rev. B 77, 155119 (2008).

${ }^{45}$ We have performed similar calculations on another type of geometry, where the molecules are bonded to the gold electrodes in a "top" position (Ref. 27). In this geometry the decay coefficients $\beta_{\mathrm{R}, \mathrm{S}, \mathrm{D}}$ are by and large the same as presented above, but the thermopowers are higher by a factor of 2 .

${ }^{46} \mathrm{We}$ obtain $Q_{\mathrm{S}}^{(0)}=2.93 \mu \mathrm{V} / \mathrm{K}, \quad Q_{\mathrm{S}}^{(1)}=4.57 \mu \mathrm{V} / \mathrm{K}$ and $Q_{\mathrm{D}}^{(0)}$ $=-16.03 \mu \mathrm{V} / \mathrm{K}, Q_{\mathrm{D}}^{(1)}=15.28 \mu \mathrm{V} / \mathrm{K}$ from best fits.

${ }^{47}$ For dimethylbenzene we average over the "meta" and "para" types.

${ }^{48}$ S. Woitellier, J. P. Launay, and C. Joachim, Chem. Phys. 131, 481 (1989).

${ }^{49}$ R. Stadler, J. Phys.: Conf. Ser. 61, 1097 (2007).

${ }^{50}$ Y. Xue and M. A. Ratner, Phys. Rev. B 68, 115407 (2003). 